\title{
Oral health-related quality of life and malnutrition in patients treated for oral cancer
}

\author{
Rocío Barrios • Georgios Tsakos • Blas García-Medina • \\ Ildefonso Martínez-Lara • Manuel Bravo
}

Received: 4 December 2013 / Accepted: 6 May 2014 / Published online: 22 May 2014

(C) The Author(s) 2014. This article is published with open access at Springerlink.com

\begin{abstract}
Purpose This study examined whether oral health-related quality of life (OHRQoL) is associated with nutritional status in patients treated for oral cancer.

Methods A cross-sectional study was carried out on with patients treated for oral cancer at least 6 months after treatment. OHRQoL was measured using two questionnaires: Oral Impacts on Daily Performances (OIDP) and Oral Health Impact Profile (OHIP-14); malnutrition risk was assessed through the Mini Nutritional Assessment (MNA). Multivariable regression models assessed the association between the outcomes (OIDP and OHIP-14) and the exposure (MNA), adjusting for sex, age, clinical stage, social class, date of treatment completion, and functional tooth units.
\end{abstract}

R. Barrios $\cdot$ M. Bravo

School of Dentistry, University of Granada, Campus Universitario

'La Cartuja' s/n, 18071 Granada, Spain

M. Bravo

e-mail: mbravo@ugr.es

G. Tsakos

Department of Epidemiology and Public Health, Institute of

Epidemiology and Health, University College London, 1-19

Torrington Place, London WC1E6BT, UK

e-mail: g.tsakos@ucl.ac.uk

B. García-Medina • I. Martínez-Lara

Servicio de Cirugía Maxilofacial, Hospital Universitario "Virgen de las Nieves", Avenida de las Fuerzas Armadas, 2, 18014 Granada, Spain

B. García-Medina

e-mail: blasmaxilo@yahoo.es

I. Martínez-Lara

e-mail: imartinezlara@yahoo.es

R. Barrios $(\bowtie)$

Calle Llanete del Mercado no. 5, 23680 Alcalá la Real, Jaen, Spain

e-mail: rbarrios@ugr.es
Results The final simple included 133 patients, $22.6 \%$ of which were malnourished or at risk of malnutrition. More than $95 \%$ of patients reported a negative impact on the OHRQoL for both measures used. Patients with malnutrition or risk of malnutrition had significantly worse OHRQoL than those with no malnutrition, even after adjusting for clinical and socioeconomic data $(\beta$-coefficient $=8.37(95 \%$ confidence interval (CI) $1.42-15.32$ ) with the OIDP and $\beta$-coefficient $=2.08$ (95\% CI 0.70-3.46) with the OHIP-14).

Conclusion Being malnourished or at risk of malnutrition is an important longer-term determinant of worse OHRQoL among patients treated for oral cancer.

Keywords Oral cancer · Malnutrition · Quality of life OHIP · OIDP · MNA

\section{Introduction}

Oral cancer is the most common of head neck cancers worldwide with approximately 263,000 new cases every year [1]. The vast majority of oral cancers consist of squamous cell carcinomas (SCC). This cancer and its treatment produce important changes in the oral cavity which may impact in basic functions, such as speech, swallowing, chewing, or salivation. The patient's quality of life can be significantly impaired by these functions [2-4].

The effect of oral cancer on the health-related quality of life (HRQoL) has been measured through a cancer specific relevant measure $[5,6]$. In addition, a variety of generic oral health-related quality of life (OHRQoL) measures could be useful to assess subjective perceptions of oral impacts [7]. The two generic OHRQoL measures most widely used are the Oral Health Impact Profile (OHIP-14) and the Oral Impacts on Daily Performances (OIDP). While these questionnaires are not specific to oral cancer patients, they potentially allow 
comparison with populations free from oral cancer while the oral cancer specific questionnaire, for example the University of Washington Quality of Life or The European Organisation for Research and Treatment of Cancer Quality of Life Questionnaire Core 30, would not be relevant.

One of the important and neglected consequences of oral and oropharyngeal cancer refers to malnutrition. This is defined as a state resulting from lack of uptake or intake of nutrition leading to altered body composition and diminished function [8]. Malnutrition has a negative effect on the morbidity and mortality of the patients $[9,10]$. The risk of malnutrition can be long lasting because in addition to being a consequence of the disease, the treatments used produce sequels in functions involved in the process of nutrition.

A recent systematic literature review of patients treated for head and neck cancer indicated that malnutrition is a strong predictor of HRQoL [11]. However, the studies included in that review were carried out in heterogeneous groups of patients and with a short follow-up after treatment. Furthermore, HRQoL was assessed through cancer-specific measures and none of these studies has used a generic OHRQoL questionnaire that would allow comparisons with other patient groups or the general population. Thereby, the aim of our study was to examine the association between OHRQoL and nutritional status after more than 6 months from treatment in patients treated for SCC oral and oropharyngeal cancer, using generic OHRQoL measures.

\section{Materials and methods}

\section{Patients}

The study was carried out at the Department of Maxillofacial Surgery of the Virgen de las Nieves University Hospital of Granada from January 2011 to April 2013. Inclusion criteria for participation in the study were the following: patients treated for oral or oropharyngeal cancer, at least 6 months have elapsed since treatment and the patients were free from recurrence of the disease. The study was approved by the Ethics Committee of the University of Granada and each participant signed an informed consent.

Collected data included the OHRQoL as the outcome variable, the nutrition as the main exposure variable and sex, age, clinical stage, tumor location, social class, date of treatment completion, and functional tooth units as covariates. Functional posterior tooth units were defined as pairs of occluding natural, restored, or fixed prosthetic postcanine teeth (molars $=2$ units; bicuspids $=1$ unit) [12]. Functional anterior tooth units were defined as pairs of occluding natural, restored, or fixed prosthetic precanine teeth (each tooth $=1$ unit).

Measurement of OHRQoL

OHRQoL was assessed through two widely used relevant generic measures. The Oral Health Impact Profile (OHIP-14) comprises 14 items that explore seven dimensions of impact: functional limitation, physical pain, psychological discomfort, physical disability, psychological disability, social disability, and handicap. The participants respond to each item according to the frequency of the impact on a five-point Likert scale (ranging from 0 to 4): never, hardly ever, occasionally, fairly often, and very often [13]. The simple count (OHIP-SC) scoring method was used where the dimensions and the total score were calculated by summing the number of impacts reported as occasionally, fairly often, or very often.

The Oral Impacts on Daily Performances (OIDP) index assesses the impact of oral conditions on eight daily performances: eating, speaking, cleaning teeth, carrying out major work or role, social contact, relaxing/sleeping, smiling, and emotional state. It evaluates the frequency and the severity of these impacts through Likert scales. For each performance a score is calculated by multiplying the frequency and severity scores. The sum of these performances scores is divided by the maximum possible score and multiplied by 100 to give percentage overall score. In addition, the OIDP extent was calculated as the number of performances affected by impacts, ranging from 0 to $8[14,15]$.

For both the OHIP-14 and the OIDP, a higher score indicates worse OHRQoL. The recall period for both was changed from the usual 12 or 6 months to 1 month. As participants were interviewed at least 6 months after the end of their oral cancer treatment, we used a 1-month time reference in order to avoid including the acute period of recovery, in the cases of recent treatment.

\section{Measurement of nutritional status}

For the assessment of nutritional status, the European Society for Clinical Nutrition and Metabolism recommend using the Mini Nutritional Assessment (MNA) [10]. The MNA can be divided into two parts: the short form (screening questionnaire) and the full version. The short form consists of six questions about weight loss or recent appetite, mobility, psychological stress or acute disease, neuropsychological problems, and body mass index. A score between 12 and 14 (maximum score) indicates satisfactory nutritional status, so you do not need to continue with the second part of the MNA. A screening score at or below 11 suggests possible state of malnutrition and the need to complete the full version of the MNA. This second part has 12 additional questions with a maximum score of 16 points, therefore the overall maximum 
MNA score is 30. The MNA score can be used to distinguish between three groups $[16,17]$ : those with adequate nutrition (score $\geq 24$ ), or those that only needed to complete the short form (screening questionnaire); those at risk of malnutrition (scores between 17 and 23.5); and those with malnutrition (scores under 17).

Table 1 Sample description and bivariate associations between nutritional status and socioeconomic variables in patients treated for oral cancer $(n=133)$

\begin{tabular}{|c|c|c|c|c|}
\hline Variable & $\begin{array}{l}\text { All } \\
n \text { (\%㐱) }\end{array}$ & $\begin{array}{l}\text { Normal } \\
n(\% \leftrightarrow)\end{array}$ & $\begin{array}{l}\text { Malnutrition/Risk } \\
n(\% \leftrightarrow)\end{array}$ & $p$ value \\
\hline All & $133(100)$ & $103(77.4)$ & $30(22.6)$ & \\
\hline Sex & & & & $0.138^{\mathrm{a}}$ \\
\hline Male & $84(63.2)$ & $69(82.1)$ & $15(17.9)$ & \\
\hline Female & $49(36.8)$ & $34(69.4)$ & $15(30.6)$ & \\
\hline \multicolumn{5}{|l|}{ Age (years) } \\
\hline$<55$ & $29(21.8)$ & $20(69.0)$ & $9(31.0)$ & \\
\hline $55-64$ & $34(25.6)$ & $30(88.2)$ & $4(11.8)$ & \\
\hline $65-74$ & $36(27.1)$ & $33(91.7)$ & $3(8.3)$ & \\
\hline$\geq 75$ & $34(25.6)$ & $20(58.8)$ & $14(41.2)$ & \\
\hline Mean \pm SD & & & & $0.234^{\mathrm{b}}$ \\
\hline Social class ${ }^{\mathrm{d}}$ & & & & $0.110^{\mathrm{c}}$ \\
\hline I & $7(5.3)$ & $6(85.7)$ & $1(14.3)$ & \\
\hline II & $8(6.0)$ & $7(87.5)$ & $1(12.5)$ & \\
\hline III & $12(9.0)$ & $11(91.7)$ & $1(8.3)$ & \\
\hline IV & $32(24.1)$ & $25(78.1)$ & 7 (21.9) & \\
\hline $\mathrm{V}$ & $74(55.6)$ & $54(73.0)$ & $20(27.0)$ & \\
\hline Tumor site & & & & $0.815^{\mathrm{a}}$ \\
\hline Tongue & $47(35.3)$ & $35(74.5)$ & $12(25.5)$ & \\
\hline Gingiva & $15(11.3)$ & $13(86.7)$ & $2(13.3)$ & \\
\hline Mouth floor & $15(11.3)$ & $11(73.3)$ & $4(26.7)$ & \\
\hline Oropharynx & $18(13.5)$ & $15(83.3)$ & $3(16.7)$ & \\
\hline Retromolar & $10(7.5)$ & $8(80.0)$ & $2(20.0)$ & \\
\hline Others & $28(21.1)$ & $21(75.0)$ & $7(25.0)$ & \\
\hline Cancer stage & & & & $0.336^{\mathrm{c}}$ \\
\hline I-II & $81(60.9)$ & $65(80.2)$ & $16(19.8)$ & \\
\hline III-IV & $52(39.1)$ & $38(73.1)$ & $14(26.9)$ & \\
\hline \multicolumn{5}{|l|}{ Follow-up (years) } \\
\hline $1-5$ & $85(63.9)$ & $64(75.3)$ & $21(24.7)$ & \\
\hline $6-10$ & $32(24.1)$ & $27(84.4)$ & $5(15.6)$ & \\
\hline $11-15$ & $13(9.8)$ & $11(84.6)$ & $2(15.4)$ & \\
\hline $16-20$ & $3(2.3)$ & $1(33.3)$ & $2(66.7)$ & \\
\hline Mean \pm SD & $5.0 \pm 4.2$ & $5.0 \pm 3.9$ & $5.0 \pm 5.2$ & $0.961^{\mathrm{b}}$ \\
\hline \multicolumn{5}{|l|}{ Functional tooth units } \\
\hline Anterior $($ mean $\pm \mathrm{sd})$ & $2.3 \pm 2.6$ & $2.6 \pm 2.7$ & $1.3 \pm 2.3$ & $0.020^{\mathrm{b}}$ \\
\hline Posterior (mean $\pm \mathrm{SD})$ & $2.4 \pm 3.6$ & $2.8 \pm 3.8$ & $1.1 \pm 2.8$ & $0.026^{\mathrm{b}}$ \\
\hline Treatment & & & & $0.715^{\mathrm{c}}$ \\
\hline $\mathrm{S}$ & $70(52.6)$ & $55(78.6)$ & $15(21.4)$ & \\
\hline $\mathrm{S}+\mathrm{RT}$ & $39(29.3)$ & $30(76.9)$ & $9(23.1)$ & \\
\hline $\mathrm{S}+\mathrm{RT}^{\mathrm{f}}+\mathrm{CH}$ & $24(18.0)$ & $18(75.0)$ & $6(25.0)$ & \\
\hline
\end{tabular}

$S$ surgery, $R T$ radiotherapy, $\mathrm{CH}$ chemotherapy

${ }^{a}$ Chi-square test with Yates continuity correction

${ }^{\mathrm{b}}$ Student's t test

${ }^{\mathrm{c}}$ Mann-Whitney test.

${ }^{\mathrm{d}}$ In descending order 
Statistical analysis

Statistical analysis was performed using the SPSS version 17.0 software package (SPSS Inc., Chicago, IL). Descriptive analysis was followed by bivariate associations between the covariates and MNA (dichotomized as normal vs. malnutrition/risk of malnutrition) using the appropriate test according to the type of variable (chi-squared for categorical variables, $t$-test for continuous normally distributed and Mann-Whitney for continuous skewed variables).

Finally, in order to assess the unadjusted association of OHRQoL with malnutrition, we carried out simple linear regression models with OIDP and OHIP-14 as outcomes and the dichotomous MNA as exposure. Both outcomes were normally distributed in our sample. Furthermore, multivariable lineal regression models were constructed to adjust this association for factors affecting HRQoL in oral cancer, (age, sex, clinical stage, follow-up, social class, type of treatment, functional tooth units) [4]. Categorical variables were transformed into dummy variables. The construction of the models was based on conceptual grounds rather than setting arbitrary cut-off points for $p$ values of the different associations. The level of statistical significant was set up to $p<0.05$.

Furthermore, to assess the clinical importance of the difference in OHRQoL between malnutrition risk patients (M) and nourished $(\mathrm{N})$ patients we calculated the standardized effect size [18] for both the OHIP and the OIDP.

\section{Results}

Descriptive data and bivariate associations between nutritional status and socioeconomic variables appear in Table 1. Of 133 patients, 84 were males (63.2\%), with a mean age of 65.4 (standard deviation 13.0) years. More than half of the patients belong to the lowest social class (V). The tongue is the most frequent location for the cancer, the clinical stages I-II the most prevalent, most patients underwent surgery and the mean of follow-up was 5.0 years (standard deviation 4.2). Overall,
$22.6 \%$ of patients were found in malnutrition $(2.3 \%)$ or risk of malnutrition $(20.3 \%)$. These patients had significantly fewer functional tooth units compared to the rest of patients who had normal nutrition. No other significant differences were found between patients that were well nourished and those with malnutrition or risk of malnutrition.

In relation to the OIDP, oral impacts affecting daily life were very common. Overall, $97 \%$ reported having at least one oral impact in the last month. Eating was the most prevalent impact $(83.5 \%)$, followed by speaking $(77.4 \%)$. The mean OIDP score was $22.4 \pm 18.2 \%$. In terms of the extent of oral impacts, $12.8 \%$ reported one impact, $15.8 \%$ two impacts, $19.5 \%$ three impacts, $12.8 \%$ four impacts and $36.1 \%$ reported from five to eight impacts (Table 2).

In terms of the OHIP-14, $95.5 \%$ of patients reported problems occasionally, fairly often, or very often. In the last month, $16.6 \%$ of patients experienced one or two impacts, $23.3 \%$ three or four and $55.6 \%$ reported five or more impacts. Physical pain, functional limitation, and physical disability were the dimensions with the higher impact (Table 3).

Patients with malnutrition or risk of malnutrition had worse OHRQoL than those with adequate nutrition; $\beta$-coefficient $=$ 12.23 (95\% confidence interval (CI) 5.03-19.42) with the OIDP and $\beta$-coefficient $=2.86$ (95\% CI 1.44-4.28) with the OHIP-14 in the unadjusted models. In the adjusted models, the respective estimates were lower but still significant; $\beta$ coefficient $=8.37(95 \%$ CI $1.42-15.32)$ for the OIDP and $\beta$ coefficient $=2.08(95 \%$ CI $0.70-3.46)$ for the OHIP-14 (Table 4). The OHRQoL scores were significantly lower (better OHRQoL) among males, older patients, and those with more functional tooth units while patients that had undergone radiotherapy had significantly worse OHRQoL according to OHIP-14.

In terms of the magnitude of the difference in OHRQoL between patients at risk of malnutrition and those without such risk, the effect sizes were $0.79(0.36-1.15)$ for OHIP (mean \pm $\mathrm{SD}=25.70 \pm 11.22$ in $\mathrm{M}$ group, and $16.80 \pm 11.24$ in $\mathrm{N}$ group) and $0.70(0.28-1.12)$ for OIDP (mean $\pm \mathrm{SD}=31.90 \pm 17.99$ in $\mathrm{M}$ group, and $19.67 \pm 17.40$ in $\mathrm{N}$ group).

Table 2 Prevalence, overall Oral Impacts on Daily Performances (OIDP) score and extent of oral impacts in patients treated for oral cancer ( $n=133$ )

\begin{tabular}{|c|c|c|c|c|c|c|c|c|c|}
\hline Statistics & Overall & Eating & Speaking & Cleaning teeth & Physical activities & Social contact & Sleeping & Smiling & Emotional status \\
\hline \multicolumn{10}{|c|}{ Prevalence of oral impacts } \\
\hline Percent & 97.0 & 83.5 & 77.4 & 14.3 & 35.3 & 35.3 & 38.3 & 24.8 & 64.7 \\
\hline \multicolumn{10}{|c|}{ Overall OIDP score } \\
\hline Mean (SD) & $22.4(18.2)$ & $9.5(7.2)$ & $7.5(6.4)$ & $1.1(3.4)$ & $2.3(4.5)$ & $3.7(6.0)$ & $2.9(4.7)$ & $2.3(5.0)$ & $6.4(6.9)$ \\
\hline \multicolumn{10}{|c|}{ Extent of impacts (number of performance with impacts) } \\
\hline Affected & 0 & 1 & 2 & 3 & 4 & 5 & 6 & 7 & 8 \\
\hline Percent & 3.0 & 12.8 & 15.8 & 19.5 & 12.8 & 8.3 & 15.0 & 10.5 & 2.3 \\
\hline
\end{tabular}


Table 3 Prevalence of oral impacts assessed with the Oral Health Impact Profile (OHIP-14) in patients treated for oral cancer $(n=133)$

\begin{tabular}{|c|c|c|c|c|c|c|c|c|}
\hline Statistics & Overall & $\begin{array}{l}\text { Functional } \\
\text { limitation }\end{array}$ & $\begin{array}{l}\text { Physical } \\
\text { pain }\end{array}$ & $\begin{array}{l}\text { Psychological } \\
\text { discomfort }\end{array}$ & $\begin{array}{l}\text { Physical } \\
\text { disability }\end{array}$ & $\begin{array}{l}\text { Mental } \\
\text { disability }\end{array}$ & $\begin{array}{l}\text { Social } \\
\text { disability }\end{array}$ & Handicap \\
\hline \multicolumn{9}{|c|}{ Number f oral impacts } \\
\hline Mean \pm SD & $5.8 \pm 3.7$ & $1.1 \pm 0.7$ & $1.2 \pm 0.8$ & $0.8 \pm 0.8$ & $1.0 \pm 0.9$ & $0.5 \pm 0.8$ & $0.5 \pm 0.7$ & $0.7 \pm 0.8$ \\
\hline \multicolumn{9}{|c|}{ Extent of impacts (number of performance with impacts) } \\
\hline Affected & 0 & $1-2$ & $3-4$ & $5-14$ & & & & \\
\hline$n(\%)$ & $6(4.5)$ & $22(16.6)$ & $31(23.3)$ & $74(55.6)$ & & & & \\
\hline
\end{tabular}

${ }^{a}$ Number of items with score $\geq 2$

\section{Discussion}

To our knowledge, this is the first study that assesses the association between OHRQoL and nutritional status in patients treated for SCC oral and oropharyngeal carcinoma. Our results indicate that those patients with malnutrition or at risk of malnutrition had considerably worse OHRQoL than those with adequate nutrition. The unadjusted associations were strong and while the adjustment for age, sex, clinical stage, follow-up, social class, type of treatment, and functional tooth units resulted in an attenuation of the estimates, they remained large and significant.

Cancer-associated malnutrition has a negative impact on the patients' HRQoL due to reduced social functioning, a more negative outlook, and poorer general health [19]. After more than 6 months posttreatment, $22.6 \%$ of the patients were either malnourished or at risk of malnutrition. This may partly be due to the fact that oral and oropharyngeal resections result in lower food consumption and inappropriate nutrition that can persist over time [19]. Also, the use of radiotherapy and chemotherapy strongly affects nutrition and recovery from such adverse treatment requires a long time [20-22]. The effects of these treatments on the oral cavity lead to the loss of natural teeth. In line with other studies [23, 24], nutritional status was significantly associated with functional tooth units in our sample.

Patients treated for oral cancer have a very high prevalence of oral impacts on their daily life; more than $95 \%$ reported a negative impact on the OHRQoL for both measures used. An earlier study showed also that oral cancer is associated with high levels of oral impacts [25]. Indeed, the prevalence in our study was higher than those described in a population without oral cancer in Spain (OIDP 68.5 \%; OHIP 85.0 \%) [26]. The difference between cancer patients and the general population could be even higher because the sample for this other study was selected from a dental centre where people tend to visit when they have dental problems. Furthermore, in our study the record period was shortened to 1 month while it was 12 months in the study by Montero et al. [14], which may have resulted in higher prevalence due to inclusion of

Table 4 Multiple linear regression analysis with OIDP or OHIP as dependent variable in patients treated for oral cancer $(n=133)$

\begin{tabular}{|c|c|c|c|c|}
\hline \multirow[t]{2}{*}{ Variable } & \multicolumn{2}{|l|}{ OIDP } & \multicolumn{2}{|l|}{ OHIP } \\
\hline & ß-coefficient (95\% CI) & $p$ value & $\beta$-coefficient $(95 \% \mathrm{CI})$ & $p$ value \\
\hline MNA & $8.37(1.42-15.32)$ & 0.019 & $2.08(0.70-3.46)$ & 0.004 \\
\hline \multicolumn{5}{|l|}{ Social class } \\
\hline V vs IV & $-0.05(-7.16-7.06)$ & 0.989 & $0.07(-1.35-1.48)$ & 0.927 \\
\hline V vs III & $-4.08(-14.50-6.35)$ & 0.440 & $0.30(-1.77-2.38)$ & 0.773 \\
\hline V vs II & $-5.65(-18.13-6.84)$ & 0.373 & $-0.45(-2.93-2.03)$ & 0.720 \\
\hline V vs I & $0.23(-13.28-13.74)$ & 0.973 & $-0.01(-2.69-2.68)$ & 0.995 \\
\hline Clinical stage (I-II) & $6.09(-3.20-15.38)$ & 0.197 & $0.76(-1.08-2.61)$ & 0.415 \\
\hline Age (years) & $-0.39(-0.64-[-0.15])$ & 0.002 & $-0.07(-0.12-[-0.02])$ & 0.006 \\
\hline Sex (male) & $12.56(5.99-19.12)$ & $<0.001$ & $2.48(1.19-3.79)$ & $<0.001$ \\
\hline Follow-up (years) & $-0.08(-0.76-0.61)$ & 0.826 & $-0.08(-0.22-0.06)$ & 0.260 \\
\hline Functional tooth & $-0.67(-1.24-[-0.10])$ & 0.021 & $-0.17(-0.28-0.05)$ & 0.004 \\
\hline \multicolumn{5}{|l|}{ Treatment } \\
\hline S vs RT & $7.72(-0.86-16.29)$ & 0.077 & $1.72(0.01-3.43)$ & 0.048 \\
\hline $\mathrm{S}$ vs $\mathrm{CH}$ & $-5.86(-17.06-5.35)$ & 0.303 & $0.42(-1.80-2.65)$ & 0.708 \\
\hline
\end{tabular}

$S$ surgery, $R T$ radiotherapy, $\mathrm{CH}$ chemotherapy 
occasional impacts. The main oral impacts were related to eating and speaking, as can be directly seen in the OIDP results and the fact that the more prevalent OHIP-14 dimensions of physical pain, functional limitation, and physical disability are related to these functions. These results corroborate those from Linsen et al. [27] in which the 10 most negative assessed items were mainly associated with feeding behavior.

HRQoL in oral cancer patients is influenced by different factors, including nutrition. A compromised nutritional status can lead to lower quality of life, depression, and a change in self-image $[11,28]$. By extension OHRQoL may also be altered. We found a significant relationship between nutritional status and OHRQoL after having controlled for possible confounding factors. This is in contrast to the study of JagerWittenaar et al. [29]. This discrepancy may be due to the use of different instruments to assess both HRQoL and nutrition between the two studies.

In terms of clinical importance, the effect sizes of the differences in OHRQoL (both for OHIP and OIDP) between patients at risk of malnutrition and those without such risk were considerable. Both showed effects that were statistically significant (the $95 \%$ CIs did not include zero) and also similar. Furthermore, according to the benchmarks suggested by Cohen [30], their magnitudes are in the range between moderate and large. In this sense, the association between OHRQoL and malnutrition risk can have important clinical implications. A nutritional screening on patients after their treatment is completed would address the potential malnutrition and result in better OHRQoL, while it could also contribute to containment of the increased economic cost of malnutrition [18].

This study has some limitations. First, it is a cross-sectional study design where the patients were assessed only once. Therefore, it does not permit comparisons with their OHRQoL pretreatment and it is not possible to attribute the OHRQoL to the treatment of oral cancer alone. This research question (effect of cancer on OHRQoL would ideally be addressed through randomized clinical trials; however, this is clearly unethical as it would deny treatment to the control group. Therefore, prospective studies are the best option and such studies are recommended to confirm our findings. Second, the generic OHRQoL questionnaires may be beneficial in terms of comparisons with other non-cancer samples or the general population, but their use does not rule out the possibility that the observed oral impacts may be due to other oral conditions, not just due to oral cancer or its treatment. This could have been addressed through the additional use of a cancer-specific OHRQoL measure. The OIDP has a condition specific feature that would attribute oral impacts to specific conditions [31], oral cancer in this case, but this was not used in this study. Future research should include such cancer-specific assessment of OHRQoL. Third, this sample is very heterogeneous regarding the follow-up period, therefore not suitable for drawing critical time periods for the association between malnutrition and OHRQoL. Finally, the MNA is an established nutrition screening tool useful for accurate evaluation of the current nutritional status of the patients. But its appropriateness to identify long-term malnutrition has not been established. This would be relevant for oral cancer patients that have long-term follow-ups, such as some of our sample. A periodical re-application of the MNA would be recommended, although there is no general consensus on how often this should happen [32].

\section{Conclusion}

This study indicated that oral cancer patients with malnutrition or risk of malnutrition have significantly worse OHRQoL than patients with adequate nutrition. Paying attention to the nutrition of patients treated for oral cancer may be an important determinant of their quality of life.

Acknowledgments This study was supported by the Andalusian Research Group CTS-503. Rocio Barrios, academic training, is being supported by the postgraduate research fellowship from the Spanish Ministry of Education, Culture and Sport.

Conflict of interest All authors declared no conflict of interest.

Open Access This article is distributed under the terms of the Creative Commons Attribution Noncommercial License which permits any noncommercial use, distribution, and reproduction in any medium, provided the original author(s) and the source are credited.

\section{References}

1. Ferlay J, Shin HR, Bray F, Forman D, Mathers C, Parkin DM (2010) Cancer incidence, mortality and prevalence worldwide in 2008. IARC, International Agency for Research on Cancer, France

2. Torres-Carranza E, Infante-Cossío P, Hernández-Guisado JM, HensAumente E, Gutierrez-Pérez JL (2008) Assessment of quality of life in oral cancer. Med Oral Patol Oral Cir Bucal 13:735-741

3. Biazevic MG, Antunes JL, Togni J, de Andrade FP, de Carvalho MB, Wünsch-Filho V (2008) Immediate impact of primary surgery on health-related quality of life of hospitalized patients with oral and oropharyngeal cancer. J Oral Maxillofac Surg 66:1343-1350

4. Chandu A, Smith AC, Rogers SN (2006) Health-related quality of life in oral cancer: a review. J Oral Maxillofac Surg 64:495-502

5. Silveira AP, Gonçalves J, Sequeira T, Ribeiro C, Lopes C, Monteiro E, Pimentel FL (2010) Patient reported outcomes in head and neck cancer: selecting instruments for quality of life integration in clinical protocols. Head Neck Oncol 2:32

6. Djan R, Penington A (2013) A systematic review of questionnaires to measure the impact of appearance on quality of life for head and neck cancer patients. J Plast Reconstr Aesthet Surg 66:647-659

7. Locker D, Allen F (2007) What do measures of 'oral healthrelated quality of life' measure? Community Dent Oral Epidemiol 35:401-411 
8. Soeters PB, Reijven PL, Bokhorst-de V, van der Schueren MA, Schols JM, Halfens RJ, Meijers JM, van Gemert WG (2008) A rational approach to nutritional assessment. Clin Nutr 27:706-716

9. Jager-Wittenaar H, Dijkstra PU, Vissink A, van der Laan BF, van Oort RP, Roodenburg JL (2007) Critical weight loss in head and neck cancer patients - prevalence and risk factors at diagnosis: an explorative study. Support Care Cancer 15:1045-1050

10. van Bokhorst-de van der Schuer, van Leeuwen PA, Kuik DJ, Klop WM, Sauerwein HP, Snow GB, Quak JJ (1999) The impact of nutritional status on the prognoses of patients with advanced head and neck cancer. Cancer 86:519-527

11. Lis CG, Gupta D, Lammersfeld CA, Markman M, Vashi PG (2012) Role of nutritional status in predicting quality of life outcomes in cancer - a systematic review of the epidemiological literature. Nutr J $11: 27$

12. Hatch JP, Shinkai RS, Sakai S, Rugh JD, Paunovich ED (2001) Determinants of masticatory performance in dentate adults. Arch Oral Biol 46:641-648

13. Montero-Martín J, Bravo-Pérez M, Albaladejo-Martínez A, Hernández-Martín LA, Rosel-Gallardo EM (2009) Validation the Oral Health Impact Profile (OHIP-14sp) for adults in Spain. Med Oral Patol Oral Cir Bucal 14:44-50

14. Montero J, Bravo M, Albaladejo A (2008) Validation of two complementary oral-health related quality of life indicators (OIDP and OSS 0-10) in two qualitatively distinct samples of the Spanish population. Health Qual Life Outcomes 6:101

15. Gherunpong S, Tsakos G, Sheiham A (2004) The prevalence and severity of oral impacts on daily performances in Thai primary school children. Health Qual Life Outcomes 2:57

16. Cuervo M, García A, Ansorena D, Sánchez-Villegas A, MartínezGonzález M, Astiasarán I, Martínez J (2008) Nutritional assessment interpretation on 22,007 Spanish community-dwelling elders through the Mini Nutritional Assessment test. Public Health Nutr 12:82-90

17. Guigoz Y (2006) The Mini Nutritional Assessment (MNA ${ }^{\circledR}$ ) review of the literature - what does it tell us? J Nutr Health Aging 10:466487

18. Viechtbauer W (2007) Approximate confidence intervals for standardized effect sizes in the two-independent and two-dependent samples design. J Educ Behav Stat 32:39-60

19. Van Cutsem E, Arends J (2005) The causes and consequences of cancer-associated malnutrition. Eur J Oncol Nurs 9:51-63

20. Airoldi M, Garzaro M, Raimondo L, Pecorari G, Giordano C, Varetto A, Caldera P, Torta R (2011) Functional and psychological evaluation after flap reconstruction plus radiotherapy in oral cancer. Head Neck 33:458-468

21. Infante-Cossio P, Torres-Carranza E, Cayuela A, Hens-Aumente E, Pastor-Gaitan P, Gutierrez-Perez JL (2009) Impact of treatment on quality of life for oral and oropharyngeal carcinoma. Int J Oral Maxillofac Surg 38:1052-1058

22. Bekiroglu F, Ghazali N, Laycock R, Katre C, Lowe D, Rogers SN (2011) Adjuvant radiotherapy and health-related quality of life of patients at intermediate risk of recurrence following primary surgery for oral squamous cell carcinoma. Oral Oncol 47:967-973

23. Samnieng P, Ueno M, Shinada K, Zaitsu T, Wright FA, Kawaguchi Y (2011) Oral health status and chewing ability is related to mininutritional assessment results in an older adult population in Thailand. J Nutr Gerontol Geriatr 30:291-304

24. Adiatman M, Ueno M, Ohnuki M, Hakuta C, Shinada K, Kawaguchi Y (2013) Functional tooth units and nutritional status of older people in care homes in Indonesia. Gerodontology 30:262-269

25. Hassel AJ, Danner D, Freier K, Hofele C, Becker-Bikowski K, Engel M (2012) Oral health-related quality of life and depression/anxiety in long-term recurrence-free patients after treatment for advanced oral squamous cell cancer. J Craniomaxillofac Surg 40:99-102

26. Montero J, Yarte JM, Bravo M, López-Valverde A (2011) Oral health-related quality of life of a consecutive sample of Spanish dental patients. Med Oral Patol Oral Cir Bucal 16:810-815

27. Jager-Wittenaar H, Dijkstra PU, Vissink A, van der Laan BF, van Oort RP, Roodenburg JL (2011) Malnutrition and quality of life in patients treated for oral or oropharyngeal cancer. Head Neck 33:490 496

28. Chasen MR, Bhargava R (2009) A descriptive review of the factors contributing to nutritional compromise in patients with head and neck cancer. Support Care Cancer 17:1345-1351

29. Linsen S, Schmidt-Beer U, Fimmers R, Gruner M, Koeck B (2009) Craniomandibular pain, bite force, and oral health-related quality of life in patients with jaw resection. J Pain Symptom Manage 37:94-106

30. Cohen J (1988) Statistical power analysis for the behavioural sciences. Lawrence Erlbaum Associates, Hillside, New Jersey

31. Bernabé E, de Oliveira CM, Sheiham A, Tsakos G (2009) Comparison of the generic and condition-specific forms of the Oral Impacts on Daily Performances (OIDP) Index. J Public Health Dent 69:176-181

32. Bauer JM, Kaiser MJ, Anthony P, Guigoz Y, Sieber CC (2008) The Mini Nutritional Assessment - its history, today's practice, and future perspectives. Nutr Clin Pract 23:388-396 